\title{
Effective population size in social Hymenoptera with worker-produced males
}

\author{
Robin E. Owen* and \\ A. R. G. Owen†
}

\author{
* Department of Biological Sciences, \\ University of Calgary, Alberta, Canada T2N 1 N4. \\ $\dagger$ Department of Zoology University of Toronto, \\ Toronto, Ontario, Canada M5S 1A1.
}

In the social Hymenoptera, which have haplodiploid inheritance, a proportion, $\psi$ of the (haploid) males can be produced by the workers. It is shown that, for the special case where each laying worker produces exactly one male that survives to maturity and mates, the variance effective population size $N_{\mathrm{e}(\mathrm{v})}=(3-\psi)^{2} F M /\left(2 F+(2-\psi)^{2} M\right)$, where $F$ and $M$ are, respectively, the number of queens and males in the population. If the sex ratio is unity or female biased then $N_{e}$ is reduced if there are worker-produced males, however with male biased sex ratios $N_{e}$ is increased compared to its value with $\psi=0$. An alternative situation, in which laying workers can each produce more than one male offspring, was investigated using computer simulations. In this case worker-produced males reduce $N_{\text {e(v) }}$ regardless of sex ratio, although the effect is relatively the most weak with male biased sex ratios.

A reduction in effective population size due to worker-produced males may contribute to the generally low levels of genetic variation found in the Hymenoptera.

\section{INTRODUCTION}

The insect order Hymenoptera is characterized by a haplodiploid genetic system in which, at the phenomenological level, diploid females are derived from fertilized eggs while haploid males arise from unfertilized eggs. In actual fact sex is determined by an underlying genic mechanism (Crozier 1971). Inheritance in haplodiploid species is equivalent to sex-linked inheritance in diploid species.

The Hymenoptera are also characterized by the occurence of eusociality in which there is a reproductive division of labour. Colonies are founded by mated females (queens) who produce daughters (workers and young queens) and males. Workers do not mate, yet in many species retain their ovaries, and under certain conditions can undergo ovarian development and lay unfertilized eggs which develop into males. This production of males by workers is now recognized to be widespread in the higher eusocial Hymenoptera, such as stingless bees, honey bees, bumble bees, vespine wasps and higher ants (for a recent review see Bourke, 1988). The effect that worker-produced males have on the evolution of sociality and optimum sex ratios has received attention (Oster and Wilson, 1978; Aoki and Moody, 1981; Pamilo,
1984; Bourke, 1988). Similarly the effect of workerproduced males on deterministic aspects of hymenopteran population genetics has been investigated (Owen, 1980, 1985a, 1986).

In this paper we examine the influence that worker-produced males have on random genetic drift through effective population size, $N_{\mathrm{e}}$. There are two contrasting views in the literature on how worker-produced males might affect $N_{\mathrm{e}}$. Kerr (1975) suggests that given a constant total number of males, then $N_{\mathrm{e}}$ will increase as the proportion of males produced by workers increases. The reasoning followed is that the number of laying workers should be added to the number of queens, to give the total number of "genetically active" females (Contel and Kerr, 1976) in the population, which is then just used in Wright's (1933) standard sex-linked formula for $N_{\mathrm{e}}$. On the other hand, Crozier (1979) argues that worker-produced males will inevitably reduce effective population size, because an extra round of gametic sampling is introduced each generation and therefore the rate of random genetic drift is increased.

We first examine the simplest case where each laying worker produces exactly one male that survives to maturity and mates. We derive an expression for effective population size and confirm the results using computer simulations. In 
this case it turns out that whether worker-produced males increase or decrease $N_{\mathrm{e}}$ depends on the sex-ratio. If the sex-ratio is unity or female biased then $N_{\mathrm{e}}$ is reduced by the presence of workerproduced males, however with male biased sex ratios $N_{\mathrm{e}}$ is increased. Next we consider one alternative scenario in which laying workers produce different numbers of males that survive to maturity and mate. In this particular case we find using computer simulations, that worker-produced males reduce $N_{\mathrm{e}}$ regardless of sex ratio, although the effect is weakest with male biased sex ratios.

\section{ONE OFFSPRING PER LAYING WORKER}

The case considered here includes that of ordinary sex-linkage and of haplodiploid inheritance.

Assume that in one generation $F$ queens mate at random with $M$ (haploid) males and their offspring consist of $F$ queens, $(1-\psi) M$ males and a number of workers. Furthermore it is supposed that $\psi M$ of these workers each produce exactly one male by parthenogenesis that survives to maturity and mates. The quantity $\psi$ is the proportion of males produced by the workers which can vary from zero to one (Owen, 1980). Thus the mating population in the next generation still consists of $F$ queens and $M$ males. A population of fixed size is postulated with numbers $F$ and $M$ constant from generation to generation. Consider a single locus with alleles $A_{1}$ and $A_{2}$ at frequencies in the first generation denoted as follows:

$\begin{array}{lll} & A_{1} & A_{2} \\ \text { Queens } & p_{\mathrm{f}} & q_{\mathrm{f}} \\ \text { Workers } & p_{\mathrm{w}} & q_{\mathrm{w}} \\ \text { Queen-produced males } & p_{\mathrm{m} 1} & q_{\mathrm{m} 1} \\ \text { Worker-produced males } & p_{\mathrm{m} 2} & q_{\mathrm{m} 2} \\ \text { All males } & p_{\mathrm{m}} & q_{\mathrm{m}}\end{array}$

The actual gene frequency of $A_{1}$ among queens as determined by gene count is

$$
\begin{aligned}
F p_{\mathrm{f}}= & \text { (number of } \left.A_{1} A_{1} \text { queens }\right) \\
& +\frac{1}{2} \text { (number of } A_{1} A_{2} \text { queens), }
\end{aligned}
$$

similarly the actual gene frequency of $\boldsymbol{A}_{1}$ among queen-produced males given by

$$
p_{\mathrm{m} 1}=\frac{\text { (number of } A_{1} \text { queen-produced males) }}{(1-\psi) M} .
$$

In the next generation a queen or a worker belongs to the genotypes $A_{1} A_{1}$ and $A_{1} A_{2}$ with respective probabilities $p_{\mathrm{f}} p_{\mathrm{m}}$ and $\left(p_{\mathrm{f}} q_{\mathrm{m}}+q_{\mathrm{f}} p_{\mathrm{m}}\right)$, thus the expected values of $p_{\mathrm{f}}^{\prime}$ and $p_{\mathrm{w}}^{\prime}$ are both equal to

$$
\begin{aligned}
E p_{\mathrm{f}}^{\prime}=E p_{\mathrm{w}}^{\prime} & =p_{\mathrm{f}} p_{\mathrm{m}}+\frac{1}{2}\left(p_{\mathrm{f}} q_{\mathrm{m}}+q_{\mathrm{f}} p_{\mathrm{m}}\right) \\
& =\frac{1}{2}\left(p_{\mathrm{f}}+p_{\mathrm{m}}\right) .
\end{aligned}
$$

The conditional variance of $p_{\mathrm{f}}^{\prime}$ is $V\left(p_{f}^{\prime}\right)$, where

$$
\begin{aligned}
F V\left(p_{\mathrm{f}}^{\prime}\right) & =p_{\mathrm{f}} p_{\mathrm{m}}+\left(\frac{1}{2}\right)^{2}\left(p_{\mathrm{f}} q_{\mathrm{m}}+q_{\mathrm{f}} p_{\mathrm{m}}\right)-E\left(p_{\mathrm{f}}^{\prime}\right)^{2} \\
& =p_{\mathrm{f}} p_{\mathrm{m}}+\frac{1}{4}\left(p_{\mathrm{f}}+p_{\mathrm{m}}-2 p_{\mathrm{f}} p_{\mathrm{m}}\right)-\frac{1}{4}\left(p_{\mathrm{f}}+p_{\mathrm{m}}\right)^{2} \\
& =\frac{1}{4}\left(p_{\mathrm{f}} q_{\mathrm{f}}+p_{\mathrm{m}} q_{\mathrm{m}}\right),
\end{aligned}
$$

i.e.,

$$
V\left(p_{\mathrm{f}}^{\prime}\right)=\left(p_{\mathrm{f}} q_{\mathrm{f}}+p_{\mathrm{m}} q_{\mathrm{m}}\right) / 4 F .
$$

For queen-produced males $E p_{\mathrm{m} 1}^{\prime}=p_{\mathrm{f}}$, and the variance $V\left(p_{\mathrm{m} 1}^{\prime}\right)$ is just that of the proportion of $A_{1}$ gametes in a total of $(1-\psi) M$ gametes when each gamete has, independently of the others, probabilities $p_{\mathrm{f}}$ of being $A_{1}$ and $q_{\mathrm{f}}$ of being $A_{2}$. Thus

$$
V\left(p_{\mathrm{m} 1}^{\prime}\right)=p_{\mathrm{f}} q_{\mathrm{f}} /(1-\psi) M,
$$

the binomial variance.

The actual gene frequency of $A_{1}$ among workerproduced males equals (number of $A_{1}$ workerproduced males) $/ \psi M$. This is the proportion of $A_{1}$ genes in a sample of $\psi M$ genes obtained by randomly drawing one gene from each of the $\psi M$ workers who each give rise to a male. Each such gene has independently of the others in the sample, a probability of being $A_{1}$ equal to the expected gene frequency of $A_{1}$ among the $\psi M$ workers. This is therefore $\frac{1}{2}\left(p_{\mathrm{f}}+p_{\mathrm{m}}\right)$ as in equation (1). Moreover the number of $\psi M$ genes in the $\psi M$ males produced by workers is binomially distributed. Therefore the variance for $p_{\mathrm{m} 2}^{\prime}$ is

$$
\begin{aligned}
V\left(p_{\mathrm{m} 2}^{\prime}\right) & =\frac{1}{2}\left(\mathrm{p}_{\mathrm{f}}+p_{\mathrm{m}}\right)\left[1-\frac{1}{2}\left(p_{\mathrm{f}}+p_{\mathrm{tn}}\right)\right] / \psi M \\
& =\left[2 p_{\mathrm{f}} q_{\mathrm{f}}+2 p_{\mathrm{m}} q_{\mathrm{m}}+\left(p_{\mathrm{f}}-p_{\mathrm{m}}\right)^{2}\right] / 4 \psi M .
\end{aligned}
$$

A more formal proof of this formula, whose validity is crucial to the argument, is given in the Appendix.

The expectation is, of course,

$$
E p_{\mathrm{m} 2}^{\prime}=\frac{1}{2}\left(p_{\mathrm{f}}+p_{\mathrm{m}}\right) \text {. }
$$

The gene frequency of $A_{1}$ among all males in the next generation is

$$
p_{\mathrm{m}}^{\prime}=(1-\psi) p_{\mathrm{m} 1}^{\prime}+\psi p_{\mathrm{m} 2}^{\prime}
$$

so that

$$
\begin{aligned}
E p_{\mathrm{m}}^{\prime} & =(1-\psi) E p_{\mathrm{m} 1}^{\prime}+\psi E p_{\mathrm{m} 2}^{\prime} \\
& =\left[(2-\psi) p_{\mathrm{f}}+\psi p_{\mathrm{m}}\right] / 2 .
\end{aligned}
$$


Consequently, if we let

$$
\bar{p}=\left[(2-\psi) p_{\mathrm{f}}+p_{\mathrm{m}}\right] /(3-\psi),
$$

then

$$
\begin{aligned}
E \bar{p}^{\prime}= & \\
& {\left[\frac{1}{2}(2-\psi)\left(p_{\mathrm{f}}+p_{\mathrm{m}}\right)+\frac{1}{2}(2-\psi) p_{\mathrm{f}}+\frac{1}{2} \psi p_{\mathrm{m}}\right] /(3-\psi) } \\
& =\left[(2-\psi) p_{\mathrm{f}}+p_{\mathrm{m}}\right] /(3-\psi)=\bar{p} .
\end{aligned}
$$

Thus $\bar{p}$ is conserved in the sense of expectation; unconditional expectations satisfy

$$
E \bar{p}^{\prime}=E \bar{p}=\left[(2-\psi) p_{\mathrm{f} 0}+p_{\mathrm{m} 0}\right] /(3-\psi)=\bar{p}_{0}
$$

where $p_{\mathrm{fo}}$ and $p_{\mathrm{m} 0}$ are initial (deterministic) values and

$$
\bar{p}_{0}=\left[(2-\psi) p_{\mathrm{fo}}+p_{\mathrm{mo}}\right] /(3-\psi)
$$

Also

$$
\begin{aligned}
E\left(p_{\mathrm{f}}^{\prime}-p_{\mathrm{m}}^{\prime}\right) & =\frac{1}{2}\left(p_{\mathrm{f}}+p_{\mathrm{m}}\right)-\left(1-\frac{1}{2} \psi\right) p_{\mathrm{f}}-\frac{1}{2} \psi p_{\mathrm{m}} \\
& =\left[-\frac{1}{2}(1-\psi)\right]\left(p_{\mathrm{f}}-p_{\mathrm{m}}\right) .
\end{aligned}
$$

It follows that for unconditional expectations

$$
\left[E\left(p_{\mathrm{f}}-p_{\mathrm{m}}\right)\right]_{t}=\left[-\frac{1}{2}(1-\psi)\right]^{t}\left(p_{\mathrm{f} 0}-p_{\mathrm{m} 0}\right),
$$

so that as $t$ approaches infinity $p_{\mathrm{f}}$ and $p_{\mathrm{m}}$ both converge in probability to the same value $\bar{p}_{0}$. Therefore it can be seen that the deterministic results (Owen, 1980) hold on average, and that these results parallel those of the standard sexlinked case (Avery, 1984). (4),

The variance of $p_{m}^{\prime}$ is from equations (3) and

$$
\begin{aligned}
V\left(p_{\mathrm{m}}^{\prime}\right)= & (1-\psi)^{2} V\left(p_{\mathrm{m} 1}^{\prime}\right)+\psi^{2} V\left(p_{\mathrm{m} 2}^{\prime}\right) \\
= & (1-\psi)^{2} \frac{p_{\mathrm{f}} q_{\mathrm{f}}}{(1-\psi) M} \\
& +\frac{\psi^{2}\left[2 p_{\mathrm{f}} q_{\mathrm{f}}+2 p_{\mathrm{m}} q_{\mathrm{m}}+\left(p_{\mathrm{f}}-p_{\mathrm{m}}\right)^{2}\right]}{4 \psi M} \\
= & \frac{(2-\psi)}{2 M} p_{\mathrm{f}} q_{\mathrm{f}}+\frac{\psi}{2 M} p_{\mathrm{m}} q_{\mathrm{m}}+\frac{\psi}{4 M}\left(p_{\mathrm{f}}-p_{\mathrm{m}}\right)^{2} .
\end{aligned}
$$

Effective population size can be obtained from the variance of the change in gene frequency per generation (Avery, 1984; Crow and Denniston, 1988). The variance of the mean gene frequency $\bar{p}^{\prime}$, conditional on the gene frequencies in the previous generation, $V\left(\bar{p}^{\prime} \mid p_{\mathrm{f}}, p_{\mathrm{m}}\right)$ is equated to $\bar{p} \bar{q} /\left(2 N_{\mathrm{e}(\mathrm{v})}\right)$ (Avery, 1984). Thus the variance effective population size (Crow and Denniston, $1988)$ is defined as

$$
N_{\mathrm{e}(\mathrm{v})}=\bar{p} \bar{q} / 2 V\left(\bar{p}^{\prime} \mid p_{\mathrm{f}}, p_{\mathrm{m}}\right) .
$$

The variance of $\bar{p}$ satisfies

$$
\begin{aligned}
(3-\psi)^{2} V\left(\bar{p}^{\prime} \mid p_{\mathrm{f}}, p_{\mathrm{m}}\right)= & (2-\psi)^{2} V\left(p_{\mathrm{f}}^{\prime}\right)+V\left(p_{\mathrm{m}}^{\prime}\right) \\
=(2-\psi)^{2} \frac{p_{\mathrm{f}} q_{\mathrm{f}}+p_{\mathrm{m}} q_{\mathrm{m}}}{4 F} & \\
& +\frac{2-\psi}{2 M} p_{\mathrm{f}} q_{\mathrm{f}} \\
& +\frac{\psi}{2 M} p_{\mathrm{m}} q_{\mathrm{m}} \\
& +\frac{\psi}{4 M}\left(p_{\mathrm{f}}-p_{\mathrm{m}}\right)^{2},
\end{aligned}
$$

as given by equations (2) and (8). At a late stage $E\left[\left(p_{\mathrm{f}}-p_{\mathrm{m}}\right)^{\prime}\right]$ is small, as therefore also $\left(p_{\mathrm{f}}-p_{\mathrm{m}}\right)$ must be, so that $p_{\mathrm{f}} \cong p_{\mathrm{m}} \cong \bar{p}$ as the deterministic and stochastic results suggest. Thus substituting in equation (10) gives,

$$
\begin{aligned}
(3-\psi)^{2} V\left(\bar{p}^{\prime} \mid p_{\mathrm{f}}, p_{\mathrm{m}}\right) \cong & (2-\psi)^{2} \frac{\bar{p} \bar{q}}{2 F} \\
& +\frac{2-\psi}{2 M} \bar{p} \bar{q}+\frac{\psi}{2 M} \bar{p} \bar{q} \\
= & \left(\frac{(2-\psi)^{2}}{2 F}+\frac{1}{M}\right) \bar{p} \bar{q}
\end{aligned}
$$

Therefore

$$
\begin{aligned}
2 V\left(\bar{p}^{\prime} \mid p_{\mathrm{f}}, p_{\mathrm{m}}\right) / \bar{p} \bar{q} & \cong\left(\frac{2-\psi)^{2}}{2 F}+\frac{1}{M}\right) \frac{2}{(3-\psi)^{2}} \\
& =\frac{2 F+(2-\psi)^{2} M}{F M(3-\psi)^{2}} .
\end{aligned}
$$

Thus, using equation (9), gives

$$
N_{\mathrm{e}(\mathrm{v})}=\frac{(3-\psi)^{2} F M}{2 F+(2-\psi)^{2} M},
$$

which reduces correctly to $9 F M / 2(F+2 M)$ when $\psi=0$, this being Wright's (1933) result, derived by the method of path coefficients.

We do not give the (fairly extensive) calculations here, however we have verified that the expression in equation (11) is the correct approximation with large $F$ and $M$ to the value obtained for the effective population size with respect to rate of approach to gene fixation, called "inbreeding effective population size" in Avery (1984) and Crow and Denniston (1988). This is done by forming iterative equations for the three quantities $E\left(p_{\mathrm{f}}, q_{\mathrm{f}}\right), E\left(p_{\mathrm{m}}, q_{\mathrm{m}}\right)$ and $E\left[\left(p_{\mathrm{f}}-p_{\mathrm{m}}\right)^{2}\right]$. Moreover our expression is also the correct approximation to the value for effective population size defined 
with respect to mutation. We have obtained exact solutions for each of the above problems. For $\psi=0$ our exact equation relating to effective population size in respect to gene fixation reduces correctly to that given in Avery (1984); his equation (4.5), page 331. It also satisfies other tests relating to situations where $F$ and $M$ are small. Our exact solution for effective population size defined with respect to mutation applies to arbitrary values of $\psi$ and arbitrary levels of mutation, backward or forward. For $\psi=0$ and weak mutation our results reduce correctly to the approximate ones given in Nagylaki (1981) and Avery (1984).

Table 1 Examples of effective population size in social Hymenoptera with worker-produced males. Estimates of $N_{\mathrm{c}(\mathrm{v})}$ are derived from equation (11) and also from computer simulations (for details see text) and equation (13) $\left(\hat{N}_{e(v)}\right)$. Three values of $\psi$ (the proportion of workerproduced males) are considered for three sex ratios with the total population number kept constant at 200 . The value $\psi=0$ corresponds to the standard sex-linked case

\begin{tabular}{|c|c|c|c|c|c|c|}
\hline \multirow[b]{3}{*}{$\psi$} & \multicolumn{6}{|c|}{$F: M($ number $)$} \\
\hline & \multicolumn{2}{|c|}{$150: 50$} & \multicolumn{2}{|c|}{$100: 100$} & \multicolumn{2}{|c|}{$50: 150$} \\
\hline & $N_{\mathrm{e}(\mathrm{v})}$ & $\hat{N}_{\mathrm{e}(\mathrm{v})}$ & $N_{\mathrm{c}(\mathrm{v})}$ & $\hat{N}_{\mathrm{e}(\mathrm{v})}$ & $N_{e(v)}$ & $\hat{N}_{\mathrm{e}(\mathrm{v})}$ \\
\hline 0 & $135 \cdot 0$ & $130 \cdot 7$ & $150 \cdot 0$ & $155 \cdot 3$ & $96 \cdot 4$ & $92 \cdot 2$ \\
\hline 0.5 & $113 \cdot 6$ & $115 \cdot 9$ & $147 \cdot 1$ & $149 \cdot 9$ & $107 \cdot 1$ & $106 \cdot 0$ \\
\hline $1 \cdot 0$ & $85 \cdot 7$ & $93 \cdot 3$ & $133 \cdot 3$ & $130 \cdot 6$ & $120 \cdot 0$ & $116 \cdot 3$ \\
\hline
\end{tabular}

Effective population size given by equation (11) was compared to that estimated from computer simulations of random genetic drift (table 1). The variance in gene frequency over 150 replicate populations was calculated for 20 generations. In each replicate an initial gene pool was constituted with alleles $A_{1}$ and $A_{2}$ at equal frequencies of $p_{\mathrm{f}}=p_{\mathrm{m}}=0 \cdot 5$. Exactly $\psi M$ worker genotypes were formed by picking gametes (sperm and eggs) at random, and each gave rise to a single male. A single gamete $\left(A_{1}\right.$ or $\left.A_{2}\right)$ was picked at random with equal probability from heterozygous workers to derive their male offspring. Queen-produced males were picked from the gene pool with $A_{1}$ at probability $p_{\mathrm{f}}$ and $\boldsymbol{A}_{2}$ at probability $q_{\mathrm{f}}$. Young queen genotypes were formed by picking gametes at random and the new gene frequencies calculated from the genotype frequencies by gene counting. The new gene frequencies in males were calculated by combining the queen-and workerproduced males. The process was then repeated using the new frequencies $p_{\mathrm{f}}^{\prime}$ and $p_{\mathrm{m}}^{\prime}$.

It is well known that at an autosomal locus the variance in gene frequency at generation $t$, $\left(\sigma^{2} p\right)_{t} \cong p_{0} q_{0}\left(1-e^{-t / 2 N_{\mathrm{e}}}\right)$ where $p_{0}$ and $q_{0}$ are the initial gene frequencies. Now since $V\left(\bar{p}^{\prime} \mid p_{\mathrm{f}}, p_{\mathrm{m}}\right)=$ $\bar{p} \bar{q} / 2 N_{\mathrm{e}(\mathrm{v})}$ (equation 9), the following holds approximately

$$
\left(\sigma^{2} \bar{p}\right)_{t} \cong \bar{p}_{0} \bar{q}_{0}\left(1-\mathrm{e}^{-t / 2 N \mathrm{e}(\mathrm{v})}\right),
$$

where $\bar{p}_{0}$ and $\bar{q}_{0}$ are the initial mean allele frequencies. Hence effective population size can be estimated from the computer simulations as

$$
\hat{N}_{\mathrm{e}(\mathrm{v})} \cong-t /\left\{2 \ln \left[\left(\bar{p}_{0} \bar{q}_{0}-s_{p t}^{2}\right) / \bar{p}_{0} \bar{q}_{0}\right]\right\},
$$

where $s_{p t}^{2}$ is the observed variance in gene frequencies over the replicate populations. In the computer simulations $\bar{p}_{0}=\bar{q}_{0}=0.5$ and $t=20$. Effective population size was determined for three values of $\psi$ for each of three sex ratios (table 1). The estimates of $N_{\mathrm{e}(\mathrm{v})}$ derived from the formula (equation(11)) and the computer simulations are in close agreement. The effect of worker-produced males is to reduce $N_{e}$ if the sex ratio is unity or female biased, however $N_{\mathrm{e}}$ is increased, compared to its value with $\psi=0$, if the sex ratio is male biased. The proportional changes in $N_{\mathrm{e}}$ for various values of $\psi$ and different sex ratios are given in table 2. It can be seen that the proportional reduction in $N_{e}$ with female biased ratios is greater than the proportional increase with male biased ratios.

Table 2 Effective population size in social Hymenoptera as a function of sex ratio and the proportion of males produced by workers $(\psi)$. The ratio of $N_{\mathrm{e}(\mathrm{v})}$ for any particular value of $\psi$ to that when $\psi=0$ is given for each sex ratio. $N_{\text {e(v) }}$ was calculated using equation (11)

\begin{tabular}{llllllll}
\hline \multicolumn{7}{l}{ Sex Ratio $F: M$} \\
\cline { 2 - 7 }$\psi$ & $3: 1$ & $2: 1$ & $1.5: 1$ & $1: 1$ & $1: 1.5$ & $1: 2$ & $1: 3$ \\
\hline 0 & 1.00 & 1.00 & 1.00 & 1.00 & 1.00 & 1.00 & 1.00 \\
0.25 & 0.93 & 0.95 & 0.97 & 1.00 & 1.02 & 1.03 & 1.05 \\
0.50 & 0.84 & 0.89 & 0.93 & 0.98 & 1.03 & 1.07 & 1.11 \\
0.75 & 0.74 & 0.81 & 0.86 & 0.95 & 1.04 & 1.10 & 1.18 \\
1.0 & 0.63 & 0.71 & 0.78 & 0.89 & 1.02 & 1.11 & 1.24
\end{tabular}

\section{MORE THAN ONE OFFSPRING PER LAYING WORKER}

The case analyzed in the previous section represented one extreme situation in which each laying worker produced exactly one male that survived to maturity and mated. This might, of course, be a realistic representation of what occurs in nature, but at the moment we have no way of knowing whether this is true or not. A more realistic scenario would be if each laying worker produced a 
different number of males. We can envisage that there are a number of alternative ways in which this could occur. For instance each worker might produce a random number of males. However we chose to model by computer simulation a simple case in which equal numbers of workers produce either $1,2,3,4$, or 5 males. This introduces a deterministic element to the problem, but serves to illustrate very well the effect of unequal family size.

The simulations were done in the same way as described earlier except for the generation of worker-produced males. This time the genotypes of the laying workers were constituted by picking gametes at random as before, but now each worker gave rise to between one and five male offspring. Gametes from heterozygous workers were picked at random with equal probability and if these gave rise to more than one male then this random sampling was repeated the appropriate number of times. Consider as an illustration an example with an equal sex ratio of $100 \mathrm{~F}: 100 \mathrm{M}$ in the population and $\psi=0 \cdot 9$; thus 90 of the males would be workerproduced. Therefore in the simulation six workers would each give rise to one male, another six would each give rise to two males, and so on until a final six would each give rise to five males, and the genotypes of all of these 30 laying workers would have been picked at random.

The results of some simulations are given in table 3 . The estimated effective population size $\hat{N}_{\text {e(v) }}$,calculated using equation (13), is shown together with the variance in female allele

Table 3 Results of computer simulations of effective population size in social Hymenoptera where laying workers can each produce more than one male offspring (see text for details). Total population number was set at 200 and the sex ratio and total number of worker-produced males was varied. Simulations were run using 150 replicate populations. The values of mean heterozygosity $(\bar{H})$, the variance of gene frequency in females $\left(s_{p}^{2}\right)$, and $\hat{N}_{\mathrm{e}(\mathrm{v})}$ (from equation (13)) at generation 20 are given in the table

\begin{tabular}{|c|c|c|c|c|c|}
\hline $\begin{array}{l}\text { Number } \\
F: M\end{array}$ & $\begin{array}{l}\text { Number } \\
\text { of worker- } \\
\text { produced } \\
\text { males }\end{array}$ & $\psi$ & $\bar{H}$ & $s_{p}^{2}$ & $\hat{N}_{\mathrm{e}(\mathrm{v})}$ \\
\hline $100: 100$ & $\begin{array}{r}0 \\
45 \\
90\end{array}$ & $\begin{array}{l}0 \\
0 \cdot 45 \\
0 \cdot 90\end{array}$ & $\begin{array}{l}0.4730 \\
0.4634 \\
0.4354\end{array}$ & $\begin{array}{l}0.0158 \\
0.0191 \\
0.0326\end{array}$ & $\begin{array}{r}153 \cdot 5 \\
125 \cdot 9 \\
71 \cdot 6\end{array}$ \\
\hline $125: 75$ & $\begin{array}{r}0 \\
75 \\
150\end{array}$ & $\begin{array}{l}0 \\
0 \cdot 5 \\
1 \cdot 0\end{array}$ & $\begin{array}{l}0.4708 \\
0.4591 \\
0.4174\end{array}$ & $\begin{array}{l}0.0169 \\
0.0213 \\
0.0417\end{array}$ & $\begin{array}{r}143 \cdot 2 \\
112 \cdot 2 \\
54 \cdot 8\end{array}$ \\
\hline $50: 150$ & $\begin{array}{r}0 \\
30 \\
75\end{array}$ & $\begin{array}{l}0 \\
0 \cdot 4 \\
1 \cdot 0\end{array}$ & $\begin{array}{l}0.4574 \\
0.4534 \\
0.4444\end{array}$ & $\begin{array}{l}0.0237 \\
0.0254 \\
0.0295\end{array}$ & $\begin{array}{r}100 \cdot 5 \\
93 \cdot 2 \\
79 \cdot 6\end{array}$ \\
\hline
\end{tabular}

frequency and mean heterozygosity (where $H=$ $\left.p_{\mathrm{f}} q_{\mathrm{m}}+q_{\mathrm{f}} p_{\mathrm{m}}\right)$ after 20 generations. Workerproduced males in this case reduce effective population size regardless of sex ratio. However the effect is least with male biased sex ratios. Note that when the sex ratio is unity or female biased the proportional reduction in $N_{\mathrm{e}}$ is considerably greater than in the case of one male per laying worker. For example with a 1:1 sex ratio and $\psi$ $=1 \cdot 0, N_{\mathrm{e}}$ is reduced by 11 per cent when each worker produces a single male (tables 1 and 2), however when some workers produce more than one male the reduction in $N_{\mathrm{e}}$ is 29 per cent (table 3).

It is of course possible to theoretically extend the formula of equation (11) to cover all cases of variable size of families produced by queens and workers, and we have indeed done this. The algebraic foundations are however somewhat lengthy and as the main thrust of this paper is in the direction of fact finding rather than mathematical exposition, in the interests of brevity we have given preference to illustrating the problem via simulation.

\section{DISCUSSION}

In this paper we have provided the first quantitative treatment of the problem of effective population size in the social Hymenoptera that have workerproduced males. We derived an explicit formula for $N_{\mathrm{e}}$ for the special case where each laying worker produces exactly one male that survives to maturity and mates. This formula showed that worker-produced males reduce $N_{\mathrm{e}}$ when population sex ratios are either unity or female biased, but increase $N_{\mathrm{e}}$ when sex ratios are male biased. Thus a reduction in $N_{\mathrm{e}}$ is not an inevitable consequence of introducing an extra round of gametic sampling each generation. Computer simulations of a perhaps more realistic situation, where some laying workers produce more than one male offspring, showed a reduction in $N_{\mathrm{e}}$ regardless of sex-ratio. Therefore it seems reasonable to conclude that worker-produced males will generally tend to reduce effective population size rather than increase it, as argued by Crozier (1979).

We have assumed that the proportion of worker-produced males $(\psi)$ is constant from one generation to the next. In fact it may fluctuate stochastically from generation to generation, for example in species where workers produce males in orphaned colonies and the frequency of orphaning (queen loss) depends on ecological conditions. 
It is well known that when a population is subject to variation in numbers then the effective population size is approximated by the harmonic mean of the successive generation sizes (Wright, 1938). Consider now the situation where population number and sex ratio is fixed but $\psi$ varies each generation. Then applying the same principle we see that approximately,

$$
1 / \bar{N}_{\mathrm{e}}=(1 / t) \sum\left(1 / N_{\mathrm{ei}}\right)
$$

where $\vec{N}_{\mathrm{e}}$ is the harmonic mean of the effective population sizes $\left(N_{\text {eis }}\right)$ dependent on $\psi$. Since the harmonic mean tends to be dominated by the smallest terms, it follows that $N_{\mathrm{e}}$ will be further reduced by fluctuating values of $\psi$ relative to its value if there were no worker-produced males.

The change in effective population size caused by having worker-produced males can be quite substantial (table 1), although the effect tends to be more pronounced when some workers can each produce more than one male offspring. Therefore, as far as $1: 1$ and female biased sex ratios are concerned, equation (11) can be thought of as giving a minimum estimate of the reduction in $N_{\text {e(v) }}$ that will occur for any particular value of $\psi$ recorded.

The significant effect that worker-produced males can have on effective population size has a bearing on the maintenance of genetic variability in the Hymenoptera. In general Hymenoptera have significantly lower levels of genetic variation, at least at electrophoretically detectable loci, than comparable diploid insects (Berkelhammer, 1983; Graur, 1985; Owen 1985b). Gene loci in haplodiploids are predicted to have lower mean heterozygosities than loci in diploids whether selection or mutation-drift maintain variability (Avery, 1984). Variation at loci possessing selectively neutral alleles depends on random drift and mutation. $N_{\mathrm{e}}$ is lower at haplodiploid loci than comparable diploid loci (unless $F>7 M$, Crozier, 1976) and so the effect of drift is greater. Workerproduced males may, therefore, generally enhance this effect. Also $N_{\mathrm{e}}$ enters into many formulae incorporating drift, mutation and selection (see Avery, 1984) including probability of and time to fixation, which thus can be affected indirectly by worker-produced males.

Acknowledgement This study was supported by a grant to R.E.O. by the Natural Sciences and Engineering Research Council of Canada.

\section{REFERENCES}

AOKI, K. AND MOODY, M. 1981. One- and two-locus models of the origin of worker behaviour in Hymenoptera. J. Theor. Biol., 89, 449-474.

AVERY, P. J. 1984. The population genetics of haplo-diploids and $\mathrm{x}$-linked genes. Genet. Res., 44, 321-341.

BERKELHAMMER, R. C. 1983. Intraspecific genetic variation and haplodiploidy, eusociality and polygyny in the Hymenoptera. Evolution, 37, 540-545.

BOURKE, A. F. G. 1988. Worker reproduction in the higher eusocial Hymenoptera. Q. Rev. Biol., 63, 291-311.

CONTEL, E. P. B. AND KERR, W. E. 1976. Origin of males in Melipona subnitida estimated from data of an isozymic polymorphic system. Genetica, 46, 271-277.

CROW, J. F. AND DENNISTON, C. 1988. Inbreeding and variance effective population numbers. Evolution, 42, 482-495.

CROZIER, R. H. 1971. Heterozygosity and sex determination in haplo-diploidy. Amer. Nat., 105, 399-412.

CROZIER, R. H. 1976. Counter-intuitive property of effective population size. Nature, 262, 384.

CROZIER, R. H. 1979. Genetics of sociality. In Herman, H. R. (ed.) Social Insects, Volume I, Academic Press, New York, pp. 223-286.

GRAUR, D. 1985. Gene diversity in Hymenoptera. Evolution, 39, 190-199.

KERR, W. E. 1975. Evolution of the population structure in bees. Genetics, 79, 73-84.

NAGYLAKI, T. 1981. The inbreeding effective population number and the expected homozygosity for an X-linked locus. Genetics, 97, 731-737.

OSTER, G. AND WILSON, E. O. 1978. Caste and Ecology in the Social Insects. Princeton University Press, Princeton, N. J., U.S.A.

OWEN, R. E. 1980. Population genetics of social Hymenoptera with worker-produced males. Heredity, 45, 31-46.

OWEN, R. E. 1985a. The opportunity for polymorphism and genic variation in social Hymenoptera with workerproduced males. Heredity, 54, 25-36.

OWEN, R. E. 1985b. Difficulties with the interpretation of patterns of genetic variation in the eusocial Hymenoptera. Evolution, 39, 201-205.

OWEN, R. E. 1986. Colony-level selection in the social insects: single locus additive and nonadditive models. Theor. Pop. Biol., 29, 198-234.

PAMILO, P. 1984. Genetic relatedness and evolution of insect sociality. Behav. Ecol. Sociobiol., 15, 241-248.

WRIGHT, S. 1933. Inbreeding and homozygosis. Proc. Natl Acad. Sci., USA, 19, 411-420.

WRIGHT, S. 1938. Size of population and breeding structure in relation to evolution. Science, $87,430-431$.

\section{APPENDIX}

Let $m$ workers only each produce one male offspring, and $m_{1}, m_{2}, m_{3}$ be the respective numbers of such workers of genotypes $A_{1} A_{1}, A_{1} A_{2}, A_{2} A_{2}$, where $m_{1}+m_{2}+m_{3}=m$. Conditional on $p_{\mathrm{f}}$ and $p_{\mathrm{m}}$, the respective probabilities of a worker being of one of these genotypes are $\theta_{1}, \theta_{2}, \theta_{3}$, Thus the probability of the observed distribution is the multinomial term $m$ ! $\theta_{1}^{m_{1}} \theta_{2}^{m_{2}} \theta_{3}^{m_{3}} / m_{1} ! m_{2} ! m_{3}$ !. Conditional on this partition $m_{1}, m_{2}, m_{3}$ of $m$ the proba- 
bility that there will be $\left(m_{1}+m_{1}^{*}\right)$ males of genotype $A_{1}$ and $\left(m_{3}^{*}+m_{3}\right)$ males of genotype $A_{2}$ is just the binomial probability $m_{2} ! / 2^{m_{2}} m_{1}^{*} ! m_{3}^{*} !$, with $m_{1}^{*}+m_{3}^{*}=m_{2}$. Thus, conditional on $p_{\mathrm{f}}$ and $p_{\mathrm{m}}$ the probability of $m_{1}+m_{1}^{*}$ males $\mathrm{A}_{1}$ and $m_{3}^{*}+$ $m_{3}$ males $\mathrm{A}_{2}$ is just

$$
\frac{m !}{m_{1} ! m_{2} ! m_{3} !} \frac{m_{2} !}{m_{1}^{*} ! m_{3}^{*} !} \theta_{1}^{m_{1}}\left(\frac{1}{2} \theta_{2}\right)^{m_{1}^{*}}\left(\frac{1}{2} \theta_{2}\right)^{m_{3}^{*}} \theta_{3}^{m_{3}}
$$

which is the multinomial probability for four classes totalling $m$ to be distributed as $m_{1}, m_{1}^{*}$, $m_{3}^{*}, m_{3}$ when the class probabilities are $\theta_{1}, \frac{1}{2} \theta_{2}$, $\frac{1}{2} \theta_{2}, \theta_{3}$. We require the probability for the $m$ objects (i.e., genotypes) of type $A_{1}$ or $A_{2}$ to be distributed as $s_{1}, s_{2}$ where $s_{1}=m_{1}+m_{1}^{*}, s_{2}=$ $m_{3}^{*}+m_{3}$. As the result of pooling classes in a multinominal distribution is a multinominal with an appropriately reduced number of classes, it follows that $s_{1}=m_{1}+m_{1}^{*}$ is distributed as a binomial variate with index $m$ and parameters $\theta_{1}+\frac{1}{2} \theta_{2}=\frac{1}{2}\left(p_{\mathrm{f}}+p_{\mathrm{m}}\right)$, which was to be proved.

If only variances are required without knowledge of the probability distributions, they can be calculated as follows. Thus for parthenogenetically produced haploid males (whether from queens or workers) we can set up the table in which $g_{1}$ is the number of $A_{1}$ genes in a single male offspring;

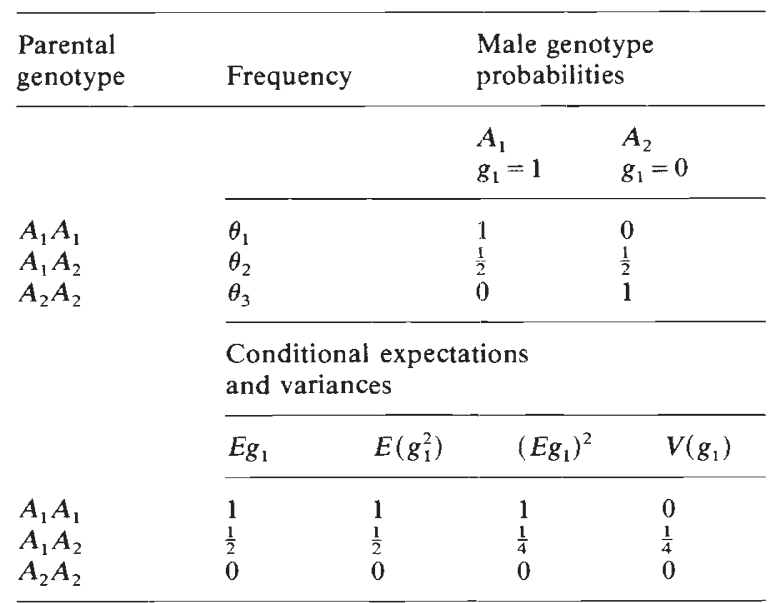

Thus $E(g)=E\left(g^{2}\right)=\theta_{1}+\frac{1}{2} \theta_{2},(E g)^{2}=\left(\theta_{1}+\frac{1}{2} \theta_{2}\right)^{2}$ so that $V(g)=\theta_{1}+\frac{1}{2} \theta_{2}-\left(\theta_{1}-\frac{1}{2} \theta_{2}\right)^{2}=p_{\mathrm{f}} q_{\mathrm{f}}$ for queenproduced males $=\frac{1}{2}\left(p_{\mathrm{f}}+p_{\mathrm{m}}\right)\left[1-\frac{1}{2}\left(p_{\mathrm{f}}+p_{\mathrm{m}}\right)\right]$ for worker-produced males, because $\theta_{1}+\frac{1}{2} \theta_{2}=$ $p_{\mathrm{f}} p_{\mathrm{m}}+\frac{1}{2}\left(p_{\mathrm{f}} q_{\mathrm{m}}+q_{\mathrm{f}} p_{\mathrm{m}}\right)=\frac{1}{2}\left(p_{\mathrm{f}}+p_{\mathrm{m}}\right)$. The required variances follow on division by the appropriate divisors, $(1-\psi) M$ for queen-produced males and $\psi M$ for worker-produced males. 